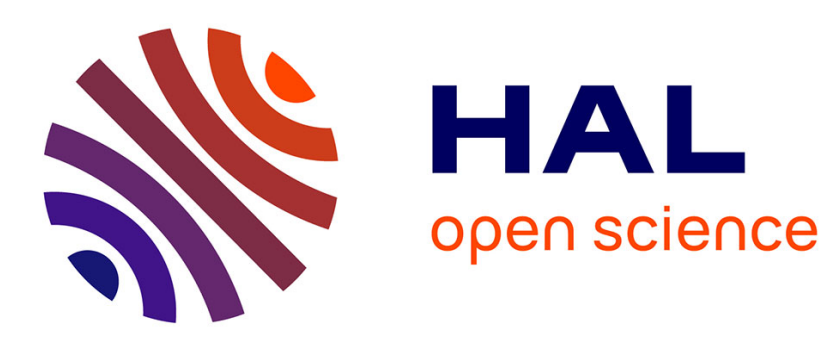

\title{
Spatial Modulation applied to Optical Wireless Communications in Indoor LOS Environments
}

\author{
Thilo Fath, Harald Haas, Marco Di Renzo, Raed Mesleh
}

\section{To cite this version:}

Thilo Fath, Harald Haas, Marco Di Renzo, Raed Mesleh. Spatial Modulation applied to Optical Wireless Communications in Indoor LOS Environments. GLOBECOM 2011, Dec 2011, Houston, United States. pp.1-6. hal-00661335

\section{HAL Id: hal-00661335 \\ https://hal-centralesupelec.archives-ouvertes.fr/hal-00661335}

Submitted on 19 Jan 2012

HAL is a multi-disciplinary open access archive for the deposit and dissemination of scientific research documents, whether they are published or not. The documents may come from teaching and research institutions in France or abroad, or from public or private research centers.
L'archive ouverte pluridisciplinaire HAL, est destinée au dépôt et à la diffusion de documents scientifiques de niveau recherche, publiés ou non, émanant des établissements d'enseignement et de recherche français ou étrangers, des laboratoires publics ou privés. 


\title{
Spatial Modulation applied to Optical Wireless Communications in Indoor LOS Environments
}

\author{
Thilo Fath*†, Harald Haas ${ }^{\dagger \S}$, Marco Di Renzo ${ }^{\ddagger}$ and Raed Mesleh ${ }^{\S}$ \\ ${ }^{*}$ EADS Innovation Works \\ Germany \\ EADS Deutschland GmbH \\ 81663 Munich, Germany \\ thilo.fath@eads.net \\ ${ }^{\dagger}$ School of Engineering and \\ Electronics \\ The University of Edinburgh \\ EH9 3JL, Edinburgh, UK \\ h.haas@ed.ac.uk \\ ${ }^{\ddagger}$ Laboratory of Signals and \\ Systems \\ French National Center for \\ Scientific Research (CNRS) \\ 91192 Gif-sur-Yvette, France \\ marco.direnzo@1ss.supelec.fr \\ $\S$ School of Engineering and \\ Science \\ Jacobs University \\ 28759 Bremen, Germany \\ r.mesleh@jacobs-university.de
}

\begin{abstract}
In this paper, we study the performance of Spatial Modulation (SM) applied to optical wireless communications (OWC) in indoor environments with line-of-sight (LOS) characteristics. To this end, we consider setup scenarios with different numbers of optical transmitters and receivers which are arranged within a room. SM is compared to repetition coding (RC). Because $\mathrm{RC}$ is known to achieve very good performance in OWC systems due to the use of intensity modulation and the resulting constructive superposition of the power signals. The results show that $\mathrm{SM}$ can outperform $\mathrm{RC}$ when high spectral efficiencies are desirable, e.g. $4 \mathrm{bit} / \mathrm{s} / \mathrm{Hz}$ and greater, since it can operate with reduced signal modulation orders by conveying additional data bits in the spatial domain. We also demonstrate that SM benefits from receive-diversity to a larger extent while at the same time requiring less computational complexity. Furthermore, we give a general framework to numerically approximate the average bit error probability of both SM and RC.
\end{abstract}

Index Terms - optical wireless communications, modulation, diversity, SM, repetition coding.

\section{INTRODUCTION}

The first pioneering studies in optical free-space transmissions for indoor environments [1] have shown the potential of optical wireless communications (OWC) to provide flexible and efficient indoor data transmission. With the advent of cheap and powerful light emitting diodes (LEDs), appropriate optical transmitters are available which can be used e.g. in home and office scenarios. As OWC transmission does not interfere with delicate electronic systems, it can even be applied to sensitive environments like hospitals and aircrafts. However, like all wireless communication systems, OWC also has to cope with the ever increasing demand for higher data rates. Therefore, it is important to provide high spectral efficiencies at low error ratios.

Commonly, OWC transmission schemes employ intensity modulation of the optical carrier and direct detection at the receiver side [2], [3]. To this end, up- and down-conversion of the signals can be done by low-cost diodes without the need for sophisticated high-frequency circuit designs. Most optical wireless links use simple modulation techniques like on-offkeying (OOK) or pulse amplitude modulation (PAM) because they offer easy implementation. PAM provides an enhanced spectral efficiency by using several intensity levels of the optical signal in contrast to OOK which encodes information by simply switching the device on and off. However, the provision of enhanced spectral efficiencies by using larger constellation sizes leads to worse bit error ratio (BER) performance. Thus, the main drawback of simple single-input-single-output (SISO) systems is that the achievable data rate at reasonable BER performance is low since a high order modulation scheme is needed. A known solution to improve the error performance of wireless communication systems is by exploitation of diversity. The reliability can be enhanced by using several receiving devices, e.g. photo diodes, which enables receive-diversity. These so called single-input-multiple-output (SIMO) methods receive several replicas of the same transmitted signal. Hence, an enlarged portion of the emitted power can be collected and the multiple signal receptions can be combined to improve the quality of the wireless link. Besides, it is possible to use several transmit devices as well. In order to provide transmitdiversity, repetition coding (RC) can be used which works by the principle that the same information is sent from multiple transmitters simultaneously. RC is known to achieve very good performance in free-space optical communications with lineof-sight (LOS) as the intensities coming from the multiple transmitters constructively add up at the receiver side [4], [5]. Because of this, RC can outperform orthogonal space-time block codes (OSTBCs) and even SIMO transmission [4].

In [6], [7] a new and promising modulation technique called Spatial Modulation (SM) has been proposed. It has been shown that SM can achieve high data rates while providing good BER performance and low system complexity. Like RC, SM employs several emitters for transmitting data. As opposed to common modulation techniques where information is conveyed by modulating the signal, SM additionally conveys data bits in the spatial domain. In order to accomplish this, SM considers the transmitter array as an additional (spatial) constellation diagram. Unlike RC, SM works by the principle that only one transmitter is active at any time instance. In addition to modulating the signal, e.g. modulation of the signal amplitude, information is also encoded in the index of the 
transmitter which emits the signal. Therefore, high spectral efficiencies can be achieved which depend not only on the signal constellation size, but also on the number of transmitters. This leads to a degree of freedom as both parameters can be traded off against each other. Furthermore, SM can deal with high channel correlation and power imbalances of wireless links [8]. As these are characteristics of optical wireless links [9], [10], SM seems to be especially suitable for OWC.

Hence, in this paper, we apply SM to OWC in indoor LOS environments. We compare SM and RC transmission with regard to their BER performance for different spectral efficiencies. Setup scenarios with different numbers of optical transmitters and receivers are considered. Furthermore, we give a numerical framework to calculate the respective average bit error probability (ABEP) of both schemes.

The remainder of the paper is organized as follows: In Section II we introduce the system model and the notations. Section III presents the optical wireless setup from which we derive the channel coefficients for the considered indoor scenarios. The BER performance of SM and RC is studied in Section IV, where numerical and simulation results are shown. Finally, Section V concludes the paper.

\section{SySTEM MODEL}

The following notations are used throughout the paper: lower case bold symbols denote vectors and upper case bold symbols denote matrices. We use $[\cdot]^{T}$ for the transpose operator, $|\cdot|$ for the absolute value and $\|\cdot\|_{F}$ for the Frobenius norm. The signal constellation size is given by $M$ and $\mathrm{d}_{\mathrm{H}}(\cdot, \cdot)$ denotes the Hamming distance of two bit assignments. $\mathrm{Q}(x)=\frac{1}{\sqrt{2 \pi}} \int_{x}^{+\infty} \exp \left(\frac{-t^{2}}{2}\right) d t$ is the Q-function. $N_{t}$ is the number of transmitters and $N_{r}$ is the number of receivers, where $n_{t}$ is the transmitter index and $n_{r}$ is the receiver index. We assume perfect knowledge of the channel and ideal time synchronisation at the receiver side. Channel coding is not taken into account.

The received signal vector is given by:

$$
\mathbf{y}=\mathbf{H} \mathbf{x}+\mathbf{n},
$$

where $\mathbf{x}=\left[\begin{array}{lll}x_{1} & \ldots & x_{N_{t}}\end{array}\right]^{T}$ is the signal vector to be transmitted. $\mathbf{H}$ is the $N_{r} \times N_{t}$ channel matrix. Its single elements $h_{n_{r} n_{t}}$ represent the respective channel coefficient of the wireless link between transmitter $n_{t}$ and receiver $n_{r}$. Furthermore, $\mathbf{n}$ is the noise, which we assume as zero mean additive white Gaussian noise (AWGN) of power $E_{n}$. The detection at the receiver is based on the maximum-likelihood (ML) principle. The ML detector decides for the signal vector $\widehat{\mathbf{x}}$ which minimises the Euclidean distance between the actual received signal $\mathbf{y}$ and all potential received signals leading to

$$
\widehat{\mathbf{x}}=\arg \max _{\mathbf{x}} p_{\mathbf{y}}(\mathbf{y} \mid \mathbf{x}, \mathbf{H})=\arg \min _{\mathbf{x}}\|\mathbf{y}-\mathbf{H} \mathbf{x}\|_{\mathrm{F}}^{2},
$$

where $p_{\mathbf{y}}$ is the probability density function of $\mathbf{y}$ conditioned on $\mathbf{x}$ and $\mathbf{H}$.

The intensities $I_{m}^{\mathrm{PAM}}$ which can be used for signal modulation applying $M-\mathrm{PAM}$ are given by

$$
I_{m}^{\mathrm{PAM}}=\frac{2 I}{M-1} m \text { for } m=0,1, \ldots,(M-1),
$$

with $I$ being the mean optical intensity emitted. Hence, $M$-PAM provides a spectral efficiency of $\log _{2}(M) \mathrm{bit} / \mathrm{s} / \mathrm{Hz}$. As RC works by the principle that all transmitters emit the same signal, $x_{1}=x_{2}=\ldots=x_{N_{t}}$ holds. In order to ensure comparability, the mean optical power transmitted has to be fixed, irrespective of the number of transmitters. Thus, for RC transmission, the optical power is equally distributed across all $N_{t}$ emitters and the intensities given in (3) have to be divided by factor $N_{t}$. In (4) we denote the ABEP of RC employing $M$-PAM for an arbitrary $N_{t} \times N_{r}$ scenario. It is a generalization of the common $M$-PAM ABEP, which is e.g. given in [2]. At this end, $E_{s}=(r I)^{2}$ is the mean electrical energy of the intensity modulated optical signal, with $r$ being the optical-to-electrical conversion coefficient. Because in intensity modulated optical communications the electrical energy is proportional to the square of the optical power [3].

By using SM, the bit sequence to be transmitted is passed to the SM encoder. As illustrated in Fig. 1, the encoder maps the bits to the signal vector $\mathbf{x}$. At any given time instance, only one transmitter radiates optical power. This means that only one element of $\mathrm{x}$ is non-zero. The index of this respective element states the index of the transmitter (e.g. LED) which is activated. Therefore, the emitter index depends on the bit sequence at the encoder input. In this way, one part of the data is transmitted. The other part of the data is conveyed in a conventional way via PAM. This means that the nonzero element of $\mathbf{x}$ is a specific signal constellation point with intensity $I_{m}$ representing additional information bits. $b_{m n_{t}}$ is the bit assignment of the signal when intensity $I_{m}$ is emitted from transmitter $n_{t}$. However, classical PAM must be modified because a signal with $I_{m}=0$ cannot be used for conveying information as in this case all elements of $\mathbf{x}$ would be zero and the spatial information would be lost. As a consequence, the intensities $I_{m}^{\mathrm{SM}}$ which can be used for SM are

$$
I_{m}^{\mathrm{SM}}=\frac{2 I}{M+1} m \text { for } m=1,2, \ldots, M .
$$

In the example displayed in Fig. 1, the data bits are arranged in blocks of 4 bits leading to a spectral efficiency of $4 \mathrm{bit} / \mathrm{s} / \mathrm{Hz}$. The last two bits define the transmitter index and the first two ones the signal intensity assuming $M=4$. As shown, for instance the bit sequence "10 011 " corresponds to LED

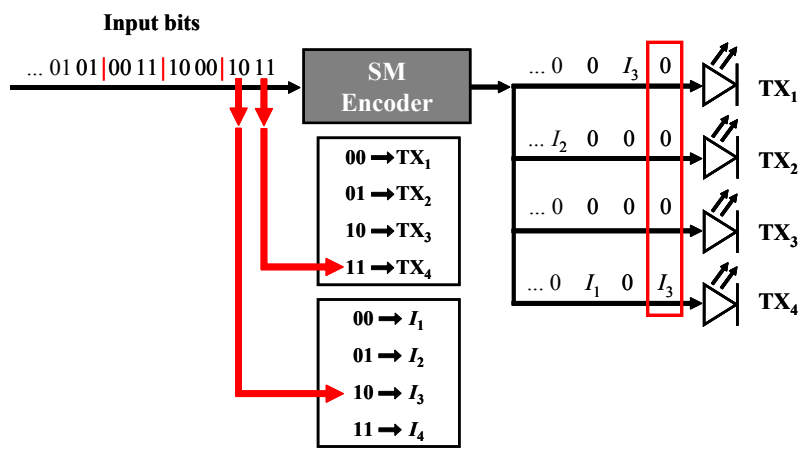

Fig. 1. Illustration of SM operation providing $4 \mathrm{bit} / \mathrm{s} / \mathrm{Hz}$ with $N_{t}=4$. 


$$
\begin{array}{r}
\mathrm{ABEP}_{\mathrm{RC}} \approx \frac{2(M-1)}{M \cdot \log _{2}(M)} \cdot \mathrm{Q}\left(\frac{1}{M-1} \cdot \sqrt{\frac{E_{s}}{E_{n} N_{t}^{2}} \cdot \sum_{n_{r}=1}^{N_{r}}\left(\sum_{n_{t}=1}^{N_{t}} h_{n_{r} n_{t}}\right)^{2}}\right) \\
\mathrm{ABEP}_{\mathrm{SM}} \leq \frac{1}{M N_{t} \cdot \log _{2}\left(M N_{t}\right)} \cdot \sum_{m^{(1)}=1}^{M} \sum_{n_{t}^{(1)}=1}^{N_{t}} \sum_{m^{(2)}=1}^{M} \sum_{n_{t}^{(2)}=1}^{N_{t}} \mathrm{~d}_{\mathrm{H}}\left(b_{m^{(1)} n_{t}^{(1)}}, b_{m^{(2)} n_{t}^{(2)}}\right) \\
\mathrm{Q}\left(\sqrt{\frac{r^{2}}{4 E_{n}} \cdot \sum_{n_{r}=1}^{N_{r}}\left|I_{m^{(2)}}^{\mathrm{SM}} \cdot h_{n_{r} n_{t}^{(2)}}-I_{m^{(1)}}^{\mathrm{SM}} \cdot h_{n_{r} n_{t}^{(1)}}\right|^{2}}\right)
\end{array}
$$

number 4 emitting an optical pulse with intensity $I_{3}$. At the receiver side, the detector has to perform two detection tasks. First, it has to estimate the index of the respective LED which is active and second it has to decode the information encoded in the signal from the received intensity level. Only if both the index and the signal constellation point are detected correctly, the bit sequence can be decoded error free. As ML detection is assumed, both estimation tasks are jointly done by the decoding algorithm given in (2).

According to (5), the minimum distance between two possible SM intensities is $\frac{2 I}{M+1}$, whereas the minimum distance for PAM is $\frac{2 I}{M-1}$. The lower signal distance of SM leads to a worse BER performance as the error probability depends on the Euclidean distance of the transmitted signals. But as SM conveys additional information bits in the spatial domain, it provides a higher spectral efficiency which is $\log _{2}(M)+\log _{2}\left(N_{t}\right)=\log _{2}\left(M N_{t}\right)$ bit/s/Hz. In other words: SM can achieve the same efficiency as $M$-PAM, but with a reduced signal constellation size of $\widetilde{M}=\frac{M}{N_{t}}$, hence effectively enlarging the distance of the signal points. In [8] and [11] it is shown that the error performance of SM depends on the differences between the channels. Moreover, it is demonstrated that the ABEP of SM can be approximated by union bound methods. Due to space constraints, we omit the detailed calculation and report only the final result for the ABEP of SM which is given in (6).

If we consider the computational complexity at the receiver side, it can be seen that the detection of SM transmission requires fewer mathematical operations compared to RC. For RC, a total of $M N_{r}\left(2 N_{t}+1\right)$ multiplications, additions and subtractions are needed. In contrast, SM merely requires $3 \widetilde{M} N_{t} N_{r}=3 M N_{r}$ operations and, therefore, is less computationally expensive. For instance, if $M=16, N_{t}=4$ and $N_{r}=4$, RC requires 576 operations, whereas SM requires only 192 operations.

\section{Optical Wireless Setup SCEnARio}

In the following, we consider an indoor LOS environment with different $N_{t} \times N_{r}$ setup scenarios. We assume that the transmitters are placed at a height of $z=2.5 \mathrm{~m}$ and are oriented downwards to point straight down from the ceiling. The receivers are located at a height of $z=0.75 \mathrm{~m}$ (e.g. height

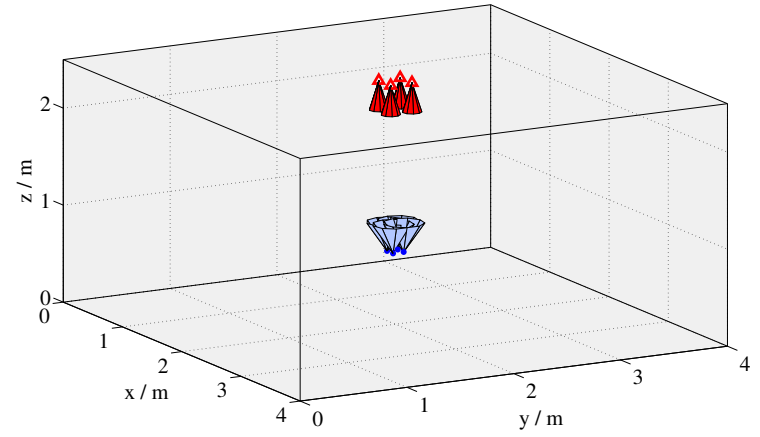

Fig. 2. Positioning of a $4 \times 4$ setup within room.

of a table) and are oriented upwards to point straight up at the ceiling. Both transmitters and receivers are aligned in rectangular arrays, which are centred within the room. The element spacing of the single transmit apertures on the $x$ - and $y$-axis is $0.2 \mathrm{~m}$ and $0.1 \mathrm{~m}$ for the receivers. Fig. 2 exemplarily shows the positioning of a $4 \times 4$ setup, at which the transmitters are displayed as triangulars and the receivers as dots. The plotted cones illustrate the orientation of the transmit beams and the orientation of the receiver field-of-view (FOV). The cone angles are related to the TX and RX semiangles.

On the basis of this setup scenario, we derive the channel coefficients of the optical wireless links. Fig. 3 illustrates the geometries used to calculate the coefficients $h_{n_{r} n_{t}}$. As shown, $\phi$ is the angle of emergence with respect to the transmitter axis and $\psi$ is the angle of incidence with respect to the receiver axis. Furthermore, $d$ depicts the distance between transmitter and receiver. According to [3], the DC channel gain is the most distinctive parameter describing an optical wireless link. Therefore, the channel coefficient of a directed LOS link can

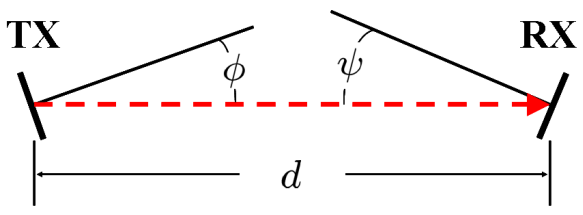

Fig. 3. Geometric scenario used for calculation of channel coefficient $h_{n_{r} n_{t}}$. 
TABLE I

VALUES OF PARAMETERS USED IN SIMULATIONS TO CALCULATE CHANNEL COEFFICIENT $h_{n_{r} n_{t}}$.

\begin{tabular}{l|l}
\hline \multicolumn{1}{c}{ Parameter } & \multicolumn{1}{c}{ Value used in simulations } \\
\hline \hline$\phi$ & $\begin{array}{l}\text { according to specific TX and RX position } \\
\text { within setup scenario, given in degree }\end{array}$ \\
\hline$\psi$ & $\begin{array}{l}\text { according to specific TX and RX position } \\
\text { within setup scenario, given in degree }\end{array}$ \\
\hline$\Phi_{\frac{1}{2}}$ & $15^{\circ}$ \\
\hline$\Psi_{\frac{1}{2}}$ & $30^{\circ}$ \\
\hline$A$ & $1 \mathrm{~cm}^{2}$ \\
\hline$r$ & $1 \mathrm{~A} / \mathrm{W}$ \\
\hline$d$ & $\begin{array}{l}\text { according to specific TX and RX position } \\
\text { within setup scenario, given in m }\end{array}$ \\
\hline
\end{tabular}

be calculated as follows:

$$
h_{n_{r} n_{t}}=\left\{\begin{array}{cl}
\frac{(k+1) A}{2 \pi d_{n_{r} n_{t}}^{2}} \cos ^{k} \phi_{n_{r} n_{t}} \cos \psi_{n_{r} n_{t}} & 0 \leq \psi_{n_{r} n_{t}} \leq \Psi_{\frac{1}{2}} \\
0 & \psi_{n_{r} n_{t}}>\Psi_{\frac{1}{2}}
\end{array}\right.
$$

with the order $k=\frac{-\ln (2)}{\ln \left(\cos \Phi_{\frac{1}{2}}\right)}$ and the transmitter semiangle $\Phi_{\frac{1}{2}}$. Furthermore, $\Psi_{\frac{1}{2}}$ denotes the FOV semiangle of the receiver and $A$ is the detector area of the receiver. Hence, the channel coefficients depend on the specific position of each transmitter and receiver within the setup scenario. Table I gives the respective values considered in the following simulations to calculate $h_{n_{r} n_{t}}$.

\section{Results on Bit ERror Ratio PerformanCE}

In this section, we study the BER performance of SM and RC using the scenario introduced in section III. We consider setups with different numbers of optical transmitters and receivers. Furthermore, we analyse the error performance for varying spectral efficiencies. In order to ensure comparability,

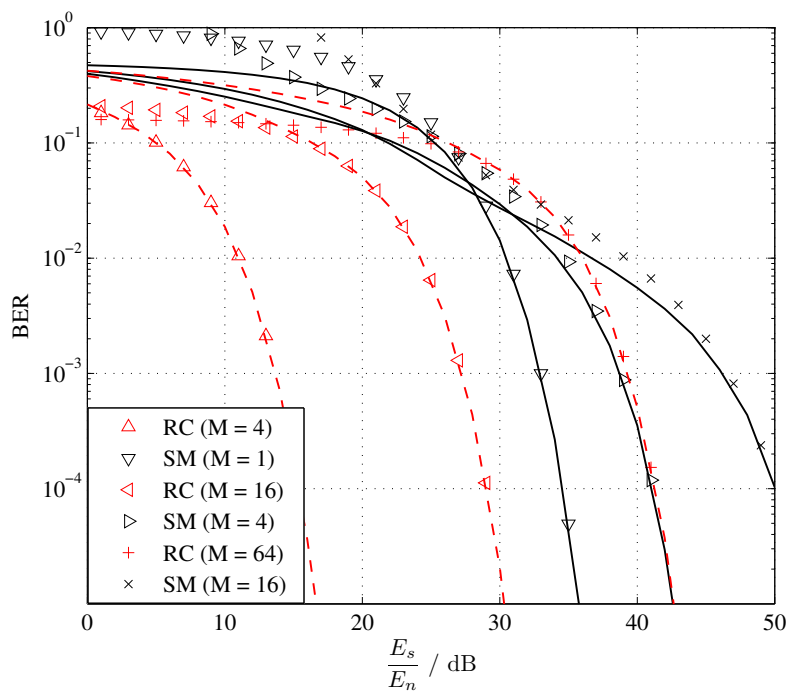

Fig. 4. Comparison of SM and RC for spectral efficiency of 2,4 and $6 \mathrm{bit} / \mathrm{s} / \mathrm{Hz}$ in $4 \times 4$ setup scenario (lines show simulation results and markers numerical ABEP results). the mean emitted power is the same in each scenario as well as for both transmission techniques.

First of all, we consider the $4 \times 4$ setup scenario illustrated in Fig. 2. For this scenario, Fig. 4 depicts the BER performance of SM and RC assuming a spectral efficiency of 2, 4 and $6 \mathrm{bit} / \mathrm{s} / \mathrm{Hz}$. It can be seen that the numerical ABEPs (markers) given in (4) and (6) very closely match the simulation results (lines). In general, the given ABEPs provide a good means to evaluate the BER performance of SM and RC because they do not depend on specific channel statistics, but only on the transfer factors. As seen in Fig. 4, for a spectral efficiency of 2 and $4 \mathrm{bit} / \mathrm{s} / \mathrm{Hz}, \mathrm{RC}$ applying 4-PAM, respectively 16-PAM, achieves a better performance than SM. But, if we consider improved spectral efficiency of $6 \mathrm{bit} / \mathrm{s} / \mathrm{Hz}, \mathrm{SM}$ outperforms RC up to a signal to noise ratio (SNR) of about $36 \mathrm{~dB}$. This is

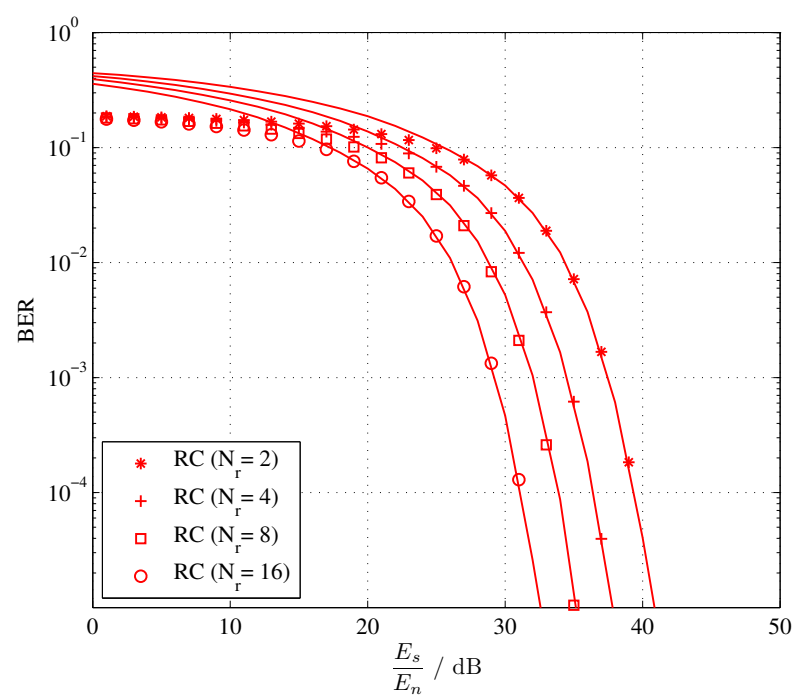

(a) 32-PAM RC

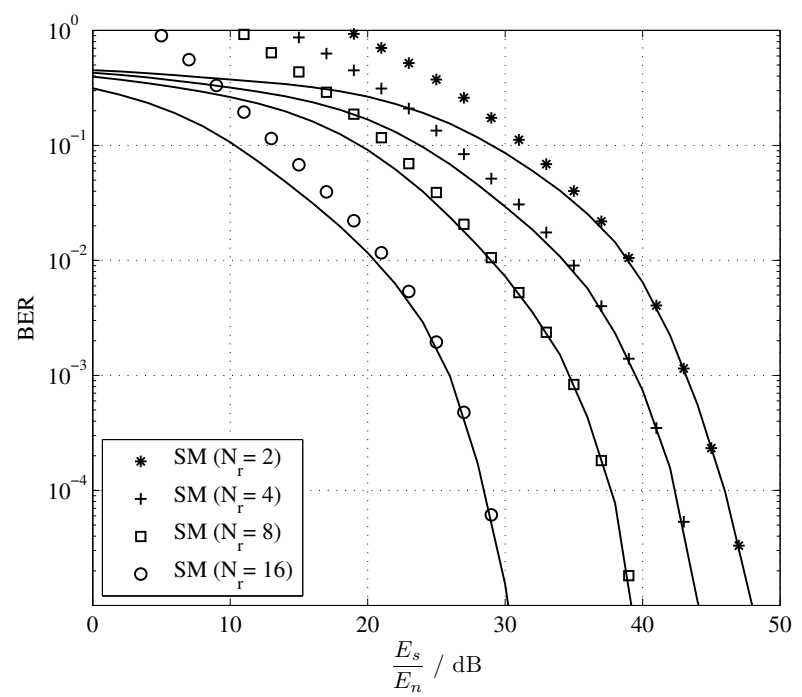

(b) SM with $M=4$

Fig. 5. Comparison of SM and RC for $5 \mathrm{bit} / \mathrm{s} / \mathrm{Hz}$ with $N_{t}=8$ and varying number of optical receivers $N_{r}$ (lines show simulation results and markers numerical ABEP results). 
because SM operates with a reduced signal constellation size of $M=16$ compared to 64-PAM RC transmission. At a SNR above $36 \mathrm{~dB}$ RC gets superior because of its transmit-diversity gain.

As SM only uses one transmitter at any time instance, it cannot provide transmit-diversity. However, it can utilize receive-diversity to a larger extent than $\mathrm{RC}$ by offering higher SNR gains with increasing $N_{r}$. This finding is shown in Fig. 5, where we study the performance of SM and RC for $5 \mathrm{bit} / \mathrm{s} / \mathrm{Hz}$ for a scenario with $N_{t}=8$ and a varying number of optical receivers. It can be seen that when consecutively doubling $N_{r}$ from 2 up to $16, \mathrm{RC}$ achieves a performance gain of about $3 \mathrm{~dB}$ in each step. In contrast, SM achieves larger improvements as it provides a performance gain of about $5 \mathrm{~dB}$ by moving from $N_{r}=4$ to $N_{r}=8$ and of about $9 \mathrm{~dB}$ by moving from $N_{r}=8$ to $N_{r}=16$. Consequently, in the $8 \times 16$ scenario SM even outperforms RC by about $2 \mathrm{~dB}$.

Besides these improvements, SM has another essential advantage over RC transmission. If more bits are to be transmitted per channel use, RC needs a higher increase in SNR to be able to provide the same BER performance. This observation is taken from Fig. 6, which shows the error ratios for a $16 \times 16$ transmission system providing different spectral efficiencies $(4,5$ and $6 \mathrm{bit} / \mathrm{s} / \mathrm{Hz})$. It can be seen that $\mathrm{RC}$ needs a SNR betterment of about $6 \mathrm{~dB}$ to achieve the same BER when providing 5 instead of $4 \mathrm{bit} / \mathrm{s} / \mathrm{Hz}$, whereas $\mathrm{SM}$ requires an increase of only $3 \mathrm{~dB}$. Consequently, SM with $M=2$ outperforms 32-PAM RC by about $8 \mathrm{~dB}$ and even 16-PAM by about $2 \mathrm{~dB}$. If the spectral efficiency is increased by 1 bit to $6 \mathrm{bit} / \mathrm{s} / \mathrm{Hz}, \mathrm{RC}$ requires additional $6 \mathrm{~dB}$, in contrast to $\mathrm{SM}$ which needs only an increase of about $4 \mathrm{~dB}$. In summary, for a spectral efficiency of $6 \mathrm{bit} / \mathrm{s} / \mathrm{Hz} \mathrm{SM}$ outperforms RC by about $10 \mathrm{~dB}$ and even outperforms the less efficient 32-PAM RC transmission by about $4 \mathrm{~dB}$. Hence, the benefits of SM over RC largely increase with greater spectral efficiencies.

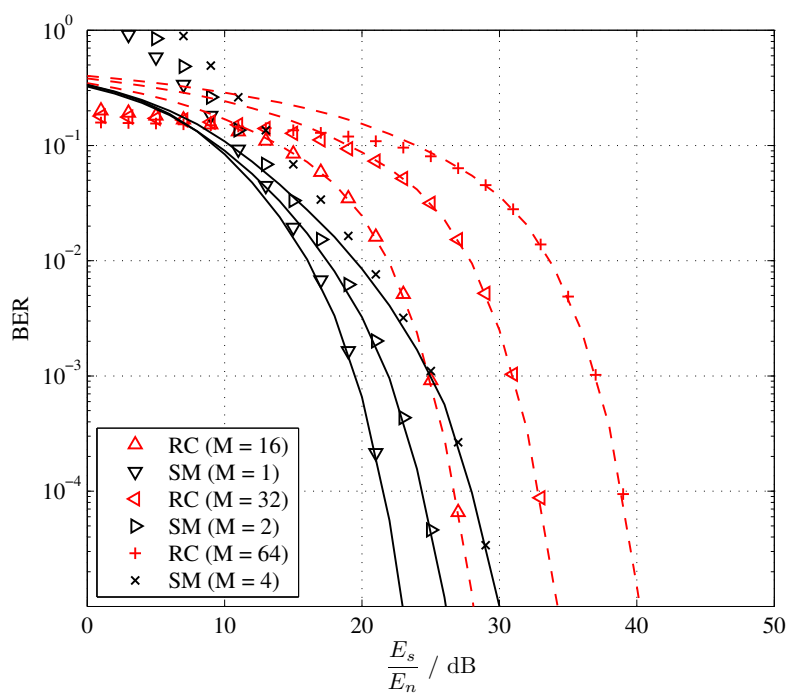

Fig. 6. Comparison of SM and RC for spectral efficiency of 4,5 and $6 \mathrm{bit} / \mathrm{s} / \mathrm{Hz}$ in $16 \times 16$ setup scenario (lines show simulation results and markers numerical ABEP results).

\section{Summary \& CONCLUSION}

We have studied the performance of Spatial Modulation applied to OWC in indoor environments and compared it to RC. The simulation results were substantiated by numerical ABEP calculations. We have demonstrated that SM achieves significant gains if several receivers are employed because it provides large SNR improvements with increasing number of receivers. Especially for higher spectral efficiencies, SM provides a better error performance as it can operate with a reduced signal constellation size by conveying data bits in the spatial domain. Furthermore, SM can achieve better error ratios particularly at low SNR regions because the transmitdiversity gain of RC prevails only at higher SNRs. Moreover, SM even provides less computational complexity. Thus, SM is a suitable modulation technique for OWC to provide high data rates at good BER performance. Future work will deal with the adaptation of SM in order to achieve benefits by transmitdiversity as this will enhance its performance, especially for low spectral efficiencies and at high SNR regions.

\section{ACKNOWLEDGEMENT}

Professor Haas acknowledges the Scottish Funding Council support of his position within the Edinburgh Research Partnership in Engineering and Mathematics between the University of Edinburgh and Heriot Watt University.

\section{REFERENCES}

[1] F. R. Gfeller and U. Bapst, "Wireless In-House Data Communication Via Diffuse Infrared Radiation," Proceedings of the IEEE, vol. 67, no. 11, pp. 1474-1486, Nov. 1979.

[2] S. Hranilovic, Wireless Optical Communication Systems, 1st ed. Springer, Sep. 1996

[3] J. M. Kahn and J. R. Barry, "Wireless Infrared Communications," Proceedings of the IEEE, vol. 85, no. 2, pp. 265-298, Feb. 1997.

[4] M. Safari and M. Uysal, "Do we really need OSTBCs for free-space optical communication with direct detection?" IEEE Transactions on Wireless Communications, vol. 7, no. 11 Part 2, pp. 4445-4448, 2008.

[5] S. M. Navidpour, M. Uysal, and M. Kavehrad, "BER Performance of Free-Space Optical Transmission with Spatial Diversity," IEEE Transactions on Wireless Communications, vol. 6, no. 8, pp. 2813-2819, Aug. 2007.

[6] Y. A. Chau and S.-H. Yu, "Space Modulation on Wireless Fading Channels," in VTC 2001 Fall Vehicular Technology Conference IEEE VTS 54th, vol. 3, 7-11 Oct. 2001, pp. 1668-1671.

[7] R. Mesleh, H. Haas, C. W. Ahn, and S. Yun, "Spatial Modulation A New Low Complexity Spectral Efficiency Enhancing Technique," in IEEE International Conference on Communication and Networking in China (CHINACOM), Beijing, China, Oct. 25-27, 2006, pp. 1-5.

[8] M. Di Renzo and H. Haas, "On the Performance of Space Shift Keying MIMO Systems Over Correlated Rician Fading Channels," in ITG International Workshop on Smart Antennas (WSA 2010), Bremen, Germany, Feb. 23-24, 2010.

[9] T. Fath, M. Di Renzo, and H. Haas, "On the Performance of Space Shift Keying for Optical Wireless Communications," in Proc. of the IEEE Global Communications Conference (GLOBECOM) - Workshop on Optical Wireless Communications, Miami, Florida, USA, Dec. 10, 2010, pp. 990-994.

[10] R. Mesleh, H. Elgala, and H. Haas, "Optical Spatial Modulation," Journal of Optical Communications and Networking, vol. 3, no. 3, Mar. 2011, (accepted, to appear).

[11] M. Di Renzo and H. Haas, "Performance Analysis of Spatial Modulation," in Proc. of the IEEE International Conference on Communications and Networking in China (CHINACOM), Beijing, China, Aug. 25-27, 2010, pp. 1-7. 